ISSN: 0213-3563

https://doi.org/10.14201/azafea2021233956

\title{
FUNDAMENTALISMO, ESTRATEGIAS ARGUMENTATIVAS Y EMOCIONES: UNA PERSPECTIVA DESDE LA COGNICIÓN DE SEGUNDO ORDEN.
}

Fundamentalism, Argumentative Strategies, and Emotions: A Perspective from Higher-Order Cognition

María Clara Garavito Gómez

Universidad Nacional de Colombia

Germán BulA

Universidad de La Salle (Colombia)

Recibido: 16 de junio de 2021

Aceptado: 17 de octubre de 2021

\section{RESUMEN}

Se examina el problema del fundamentalismo desde el punto de vista de la cognición de segundo orden, es decir, los sistemas compuestos por más de un solo individuo. Se propone que estos sistemas funcionan bien si 1) son diversos, es decir, recogen en sí diversas perspectivas y heurísticas; y 2) tienen cohesión, es decir, las diferentes partes que los componen se escuchan entre sí. El fundamentalismo se caracteriza tanto desde el punto de vista del autoritarismo en autores como Robert Altemeyer y Teodoro Adorno, como desde teorías de la cognición autoritaria y democrática, como la de Solomon Schimmel y Humberto Maturana, como de los fundamentos de la política y emociones morales de Johnatan Haidt. Este se entenderá como un esfuerzo activo, que incluye sentimientos como el odio, de no participar en el cerebro mundial, entendido como un sistema de segundo orden que incluye a todos los seres humanos.

Palabras clave: fundamentalismo; cognición colectiva; cognición corporizada; burbujas cognitivas; acrobacias pseudocognitivas; emocionar. 


\section{ABSTRACT}

This paper examines the problem of fundamentalism from the point of view of higher-order cognition; that is, of cognitive systems composed by more than one human being. It is proposed that second-order brains work well if 1) they are diverse; that is, it contains diverse perspectives and heuristics; and 2) if they have cohesion, that is if the different parts listen to each other. Fundamentalism is characterized by the idea of authoritarianism in authors such as Robert Altemeyer or Theodor Adorno, by theories of authoritarian and democratic cognition in Solomon Schimmel and Humberto Maturana, and by the idea of political foundations and moral emotions of Johnathan Haidt. Fundamentalism is understood as an active effort that involves feelings such as hate, of evading to participate in the World Brain, namely, the higher-order system that gathers to all human beings.

Key words: fundamentalism; collective cognition; Solomon Schimmel; Julius Evola; Robert Altemeyer; Theodor Adorno.

\section{INTRODUCCIÓN}

En contra de ciertas expectativas ilustradas de un progreso estable y duradero hacia una sociedad regida por la razón, asistimos en el presente al surgimiento y resurgimiento de fundamentalismos de variadas estofas. Para quienes hemos asumido la meta de participar en la construcción de sociedades diversas y pluralistas, los fundamentalismos presentan el problema de ser al mismo tiempo una manifestación y un ataque a la diferencia; ellos presentan el problema de en qué medida se debe tolerar la intolerancia (cfr. Guiora, 2014).

¿Qué se entiende por fundamentalismo? En principio, un síndrome, una colección de rasgos de personalidad que tienden a encontrarse consistentemente en los mismos individuos. Tanto Adorno et al. (2006), después de la Segunda Guerra Mundial y preocupados por la posibilidad de que los Estados Unidos sean presa del fascismo; como Altemeyer (2006), a finales del siglo XX preocupado por el poder creciente de la derecha religiosa en el partido republicano y la política estadounidense en general, encuentran que los individuos potencialmente fascistas (para Adorno), o autoritarios o de derechas (para Altemeyer) son muy respetuosos con la autoridad, convencionales (en el sentido de querer ser como la norma de su grupo de referencia), tribalistas (en el sentido de tener actitudes muy positivas hacia el grupo de referencia y muy negativas hacia grupos exteriores), presos del miedo y dispuestos a con- 
vertir el miedo en agresión cuando dicha agresión es sancionada por alguna autoridad. Como se puede ver, el fundamentalismo no es propio de algún credo en particular; inclusive se podría argumentar que, por ejemplo, ciertos defensores de la economía de mercado caen en actitudes fundamentalistas.

Para los propósitos de este texto, interesan en particular los rasgos cognitivos de este síndrome. Las personas autoritarias suelen aferrarse acríticamente a ciertos dogmas. Altemeyer caracteriza la mente autoritaria como un armario en el que se guardan diferentes creencias sin preocupación alguna porque sean coherentes entre sí $(2006,75)$; y hace notar que los autoritarios utilizan su capacidad de pensamiento crítico de manera selectiva, sólo contra aquellas ideas que amenazan sus dogmas $(2006,78)$. El psicólogo Johnathan Haidt (2003) aborda las emociones morales que están a la base del pensamiento político de derecha extrema, con lo que se reconoce que la forma de pensar de los sujetos autoritarios también incluye estrategias de difusión de emociones, especialmente asociadas al odio. En este artículo usaremos los términos fundamentalista y fundamentalismo para hablar de los hábitos cognitivos autoritarios, o de individuos potencialmente fascistas, y sus implicaciones para las cogniciones de segundo orden.

Aquí presentamos una reflexión sobre el tema desde la perspectiva de la cognición de segundo orden, es decir, la que corresponde a los grupos sociales; haciendo abstracción de las razones axiológicas por las que se debe abrigar la diversidad. Concebimos los mecanismos sociales de toma de decisiones (que incluyen la academia, la esfera pública, los mecanismos democráticos, etc.), como cerebros compuestos de cerebros, y lo que de ellos resulta es entendido como inteligencia cívica, de acuerdo con Schuler (2001). Desde ahí nos preguntamos por el papel que tienen los fundamentalismos en la salud de dichos cerebros colectivos. A manera de horizonte, tenemos en mente la noción de "cerebro mundial" ( $c f r$. Schuler, 2001), esto es, el mecanismo, en la medida que existe, que tiene la humanidad para tomar decisiones colectivas. Si bien este cerebro mundial remite a procesos cognitivos mucho más abstractos que los de segundo orden que se pueden detectar fácilmente en un grupo de trabajo, una familia, o inclusive una ciudad, consideramos que es necesario tenerlo en mente, porque hoy en día la humanidad se enfrenta a retos globales (tales como la crisis ambiental, las pandemias o la desigualdad global), que tendrá que afrontar como humanidad total.

Entender el fundamentalismo desde la comprensión de la sociedad como un sistema cognitivo tendrá diferentes retos que desarrollaremos a lo largo del artículo. Inicialmente, se responde a la pregunta: ¿Cómo estudiar esos cerebros de segundo orden? De acuerdo con el cibernético Stafford Beer 
(1995), todo sistema viable, esto es, capaz de permanecer en el tiempo gracias a su propia organización, tiene una serie de rasgos invariantes, trátese de una célula, un ser humano, una empresa o un nicho ecológico. Los sistemas viables, en tanto son capaces de adaptarse a un entorno cambiante, son ipso facto sistemas cognitivos; y, de nuevo, tienen rasgos que no varían. Por ello, es posible usar el modelo del sistema viable (VSM por sus siglas en inglés) propuesto por Beer (1995) para pensar la cognición de segundo orden; y resulta válido acudir a la analogía con el cerebro para entender los procesos cognitivos de los grupos sociales. En este texto no se aportarán argumentos directos a favor del VSM, pero esperamos que mediante el desarrollo del problema del fundamentalismo se muestre su pertinencia.

Posteriormente, y en el interés de entender el fundamentalismo en términos de esos sistemas viables, especialmente en su caracterización como un sistema cognitivo, abordaremos las nociones de "acrobacias pseudocognitivas" de Schimmel (2008) y de "burbujas cognitivas” (Bula, Garavito y González, 2021). Con estas dos nociones nos referimos a dos rasgos fundamentalistas. Al igual que un sistema cognitivo individual, el colectivo también se apropia de mecanismos que evitan la disonancia cognitiva que resulta cuando se evalúa al propio grupo con el mismo rasero que se evalúa a otros. Estos mecanismos buscan proteger una identidad cerrada, jerárquica, autocontenida y no susceptible a la autocrítica y al cambio. Al respecto, señalaremos algunas ideas sobre el efecto de tales rasgos fundamentalistas en los grupos sociales.

Finalmente, nos aproximaremos a estrategias que recientemente se entienden como cognitivas (por ejemplo, Colombetti, 2014). Nos referimos a aquellos recursos afectivos que buscan difundir ideas, principios e imaginarios, altamente efectivos en decisiones políticas. Así, se plantea que los sistemas cognitivos de segundo orden también involucran a los cuerpos individuales $\mathrm{y}$ a las experiencias intercorporales en la toma de decisiones. Y, sobre todo, planteamos que ciertas estrategias discursivas buscan, mediante la resonancia afectiva, guiar a la acción con el convencimiento de que está siendo dirigido por argumentos perfectamente racionales. Consideramos que todo sistema viable debe considerar esa dimensión emocional, siguiendo algunas perspectivas de sistemas como la de Humberto Maturana (1995).

\section{COGNICIÓN DE SEGUNDO ORDEN Y EL PROBLEMA DEL FUNDAMENTALISMO}

Haciendo a un lado consideraciones axiológicas, ¿no funcionaría mejor el cerebro social si las tareas de toma de decisiones se dejaran a los expertos? 
Según John Dewey, "Los individuos de la masa sumergida puede que no sean muy sabios, pero hay una cosa de la que saben más que cualquier otra persona, esto es, dónde les aprieta el zapato, los problemas que los hacen sufrir (traducción nuestra)"1. Este argumento se puede formular de forma más rigurosa y más general desde la cibernética: un sistema que administre a otro, debe tener una cantidad igual o mayor de variedad (esto es, la cantidad de estados posibles de un sistema) que el sistema que administra ( $c f r$. Beer, 1974, 21-22): la variedad regulativa debe alcanzar a la variedad sistémica. Cualquier cerebro humano tiene una variedad limitada por su estructura física (la cantidad combinatoria de estados posibles de sus neuronas; sin duda una cantidad muy alta pero no infinita); si dicho cerebro tiene la tarea de administrar un sistema cuya variedad lo supera (por ejemplo, una empresa, una ciudad, o el mundo), debe reducir drásticamente dicha variedad para lograr la tarea. Y las medidas autoritarias son estrategias frecuentemente usadas para restringir el comportamiento de los componentes del sistema que ha de ser administrado.

Ahora bien, se ha insistido en que una buena administración tiene que ver con la competencia. Walter Lippman critica el gobierno ciudadano porque, según él, los ciudadanos no son omnicompetentes en las materias concernientes a su propio gobierno, por lo que sería irresponsable dejar que gobiernen. Entonces necesitamos ese administrador competente (y, por ende, autoritario). Pero la crítica al gobierno ciudadano se puede volcar contra sí misma: las élites tampoco pueden ser omnicompetentes (Shuler, 2001, 162) Más bien hay que deshacerse de la idea de omnicompetencia como condición para el gobierno; la variedad regulativa necesaria se consigue a través de la participación ciudadana, de modo que el administrador no gobierna sólo, sino ayudado por los gobernados. Es de esta manera en la que podemos garantizar una administración que no implique el sacrificio de la variedad.

Obviamente, el decretar la participación no garantiza una cognición de segundo orden que sea suficiente para gobernar. Es necesario entender la arquitectura apropiada para estos procesos de segundo orden y la forma en que deben estar organizados. Para pensar dicha arquitectura volvamos a Beer: en el VSM los sistemas de primer y segundo orden están a su vez conformados por diferentes niveles de organización. No nos detendremos en todos esos niveles y cómo se aplican a una sociedad, pero detallemos uno de esos nive-

1. "The individuals of the submerged mass may not be very wise, but there is one thing they are wiser about than anybody else can be, and that is where the shoe pinches, the troubles they suffer from" (Dewey, 1950, p. 348). 
les, el del subsistema 5. Este está dedicado a pensar la identidad de un sistema viable (lo que, en individuos y colectivos humanos, tendría que ver con la pregunta ‘ ¿cómo se debe vivir?’). De acuerdo con Beer, este subsistema funciona como un multinodo (1995, 205); esto es, como una colección descentralizada de operadores cognitivos. En una sociedad, esos operadores son los individuos, con un esquema de conectividad que cambia según la historia del multinodo, es decir, que cambia con las relaciones históricas de los individuos. Ellos además tienen la capacidad de afectarse mutuamente, de modo que determinados fenómenos pueden producir un efecto de bola de nieve (unos nodos activan a otros, otros a otros más) llegando a ocupar el centro de atención del multinodo. Así describe Daniel Dennett la conciencia en el cerebro: cierto fenómeno activa un grupo de neuronas, que activan a otras y a otras más, hasta que el fenómeno gana "fama cerebral" (2001); una experiencia consciente puede empezar en cualquier parte del multinodo, siempre y cuándo un nodo -individuo o neurona- logre contagiar a los demás. Estos procesos de contagio, además, modifican la estructura de conexiones del multinodo.

Un subsistema 5 determinado por la historia de interrelaciones de los individuos, supone una arquitectura abierta cuyo espacio de problema (¿cómo se debe vivir?) es indefinido. Se puede contrastar esta arquitectura con la de funciones más básicas (por ejemplo, los reflejos incondicionados) a los que conviene una arquitectura cerrada: en principio, el problema de si hay que retirar la mano cuando tocamos algo muy caliente está resuelto, por lo que se puede instalar una maquinaria con una conectividad fija que, sencillamente, cumpla la tarea. Efectivamente, en el cerebro humano, los procesos de administración del aquí y el ahora (subsistemas 2 y 3 ) tienen estructuras más fijas y limitadas. Pero el problema de la propia identidad de un sistema implica la exploración de un espacio muy grande de posibilidades, por lo cual es deseable explorarlo mediante un multinodo grande y diverso, siempre y cuando tenga la suficiente conectividad para que esta variedad pueda aprovecharse. Para no extendernos en el modelo de Beer, resumimos estas ideas en una fórmula aproximada: el poder de la cognición (de cualquier orden) es el producto de su variedad (cantidad y diversidad de nodos) y su cohesión (qué tan conectados están esos nodos).

Scott Page (2007a, 2007b) explica desde una perspectiva matemática, enriquecida con datos empíricos, el valor de la diversidad para la cognición: para problemas suficientemente complejos, un grupo diverso de no expertos encuentra mejores soluciones que un grupo de expertos. Los expertos tienden 
a converger en sus perspectivas y heurísticas, de modo que casi da lo mismo tener un solo experto que varios, lo que los hace converger en algún óptimo local, es decir, en una solución adecuada, pero no la mejor. Por otro lado, en el grupo diverso hay múltiples perspectivas cuya interacción evita que la solución se asiente en un óptimo local, y hace más probable encontrar el óptimo global. Las diversas perspectivas no se suman de forma meramente aritmética, sino que hay superaditividad: un descubrimiento realizado mediante la heurística a puede hacer posible uno mediante la heurística $b$, que de otro modo hubiera sido inútil para el problema (Page, 2007a, 13).

Por ejemplo, el descubrimiento de la matemática que rige el comportamiento de flocado en los grupos de pájaros o peces de ciertas especies (de modo que se mueven como un todo), no fue descubierto por biólogos, sino por Craig Reynolds (1987), un experto en gráficas computarizadas que ha trabajado en películas como Tron o Batman Regresa. Por un lado, Reynolds se acercó al problema desde el punto de vista de lograr una imagen convincente; por otro, los biólogos ahora pueden aprovechar su trabajo desde su perspectiva propia. A partir de estas ideas, Page proporciona argumentos convincentes para, por ejemplo, aplicar políticas de diversidad en la contratación en empresas. Ahora bien, el mismo Page (2007b, 239 y ss) es perfectamente consciente de que las ventajas teóricas de la diversidad para la cognición colectiva sólo se hacen efectivas cuando la diversidad no amenaza la cohesión de un grupo de trabajo. Las diferencias culturales, lingüísticas, e inclusive de perspectivas y heurísticas generan su propio ruido; $y$, sobre todo, un grupo diverso también tendrá preferencias diversas, es decir, diferencias respecto a los resultados deseados. Todo esto, como cualquier persona ha experimentado alguna vez en su vida, puede hacer que un grupo compuesto incluso de personas diversas e inteligentes, pueda funcionar, como cognición de segundo orden, de forma muy estúpida. De nuevo tenemos la misma fórmula: la inteligencia de un colectivo es el producto de la diversidad y la cohesión.

Ahora bien, si la diversidad puede producir patologías, la homogeneidad no está exenta de ellas. En concreto, los grupos de expertos con perspectivas y heurísticas similares pueden caer en el groupthink, una forma de cognición sesgada por el deseo de convergencia con otros miembros del grupo. Las malas decisiones de política exterior, como la fallida invasión a la Bahía de Cochinos, o la escalada de hostilidades en Vietnam, tomadas por el expertísimo gabinete de John F. Kennedy, los llamados "best and brightest", se han atribuido al fenómeno del groupthink (Janis, 1980; Halberstam, 1993). De nuevo, podemos acudir a la arquitectura general del multinodo planteada por 
Beer: los multinodos se previenen de decisiones fallidas mediante funciones antagonistas. Esto ocurre tanto en cerebros biológicos (donde sistemas como el simpático y parasimpático funcionan como controles mutuos), como en colectivos humanos bien organizados, donde, por ejemplo, la división financiera sirve para atenuar los sueños locos de la división de planeación ( $c f r$. Beer, 1995, 110). Con una idea de la arquitectura adecuada de una cognición de segundo orden (diversidad, cohesión y funciones antagonistas) podemos pasar a examinar los rasgos fundamentalistas y su rol dentro de los sistemas de segundo orden.

\section{Primer RAsgo del Fundamentalismo: Acrobacias PSEUdocognitivas}

Schimmel caracteriza los esfuerzos cognitivos que llevan a cabo los fundamentalistas para defender sus creencias como "acrobacias pseudocognitivas" $(2008,29)$. Esto se puede ilustrar con el caso de los fundamentalistas bíblicos. El fundamentalismo bíblico norteamericano se define a sí mismo por su adherencia a tres principios, que la Biblia es 1) inerrante, en tanto palabra de Dios, 2) cierta en sentido literal, y 3) clara en su sentido para cualquier lector (los dos últimos principios siendo en buena medida una reacción a los altos estudios bíblicos venidos de Europa, más propios de un protestantismo liberal) (Schimmel, 2008, 101-102). En la práctica, 2 y 3 se sacrifican para preservar 1: por ejemplo, las contradicciones internas que rendiría una lectura literal de la Biblia se solucionan mediante el "dispensacionalismo", esto es, la idea de que Dios ha revelado su palabra de forma progresiva y conectada en múltiples dispensaciones adecuadas a diferentes periodos históricos; entonces, las contradicciones entre una y otra parte de la Biblia se resuelven diciendo que pertenecen a una dispensación diferente (Schimmel, 2008, 104) (p. ej, la diferencia entre las prescripciones del Viejo Testamento y la nueva ley de Jesús). Ahora bien, el dispensacionalismo como tal, viola claramente el principio 3, pues no hay nada en la Biblia misma que aclare que se debe leer según diferentes dispensaciones.

Resulta curioso que hoy en día muchos fundamentalistas no interpretan de forma llana el famoso pasaje del libro de Josué (10:12-15) en el que, según la lectura más conocida del mismo (que le valiera la amenaza de la hoguera a Galileo), éste detiene el Sol para prolongar una batalla y poder vencer a ciertos enemigos de los hebreos. Por el contrario, muchos fundamentalistas actuales sostienen que por "detener el Sol”, hay que entender que algún 
fenómeno meteorológico (como nubes, o una tormenta de granizo), detuvo los rayos del Sol, de modo que el brillo de este no se sintió por un tiempo, y que era la oscuridad la que convenía a los hebreos en la batalla (Schimmel, $2008,105)$. Esta lectura retorcida se puede explicar por el hecho de que, hoy en día, es muy difícil sostener en público que el Sol se mueve alrededor de la Tierra. Entonces, la defensa indirecta del principio 1 implica abandonar el principio 3 para poder defender el 2. En general, un estudio de la manera en que los fundamentalistas leen la Biblia revela que, lejos de hacer una lectura llana y literal de la Biblia, oscilan mucho entre lecturas literales y no-literales en nombre de la inerrancia Bíblica, produciendo teorías exegéticas complejas (como el ya mencionado dispensacionalismo) que demuestran, entre otras cosas, que no hay que tratar a los fundamentalistas como faltos de inteligencia, sino como resueltos a aplicarla de una forma muy particular (Schimmel, 2008, 108).

Como muestra la cambiante lectura de Josué 10:12-15, las creencias fundamentalistas no son inmóviles, sino que muestran adaptabilidad, justamente a través de las acrobacias pseudocognitivas que presentan. Vale la pena mencionar el caso de Sabbatai Zevi, un rabino judío habitante del Imperio Otomano en el siglo XVII que afirmó ser el Mesías, y logró acumular una masa sustancial de creyentes y seguidores. Después de ser capturado por las autoridades otomanas, se le dio la alternativa entre morir o convertirse al islam, y Zevi escogió lo segundo. Lejos de abandonar sus creencias, los seguidores de Zevi desarrollaron una espectacular innovación teológica: la teoría de un Mesías apóstata.

Desde el punto de vista del cerebro fundamentalista, las acrobacias pseudocognitivas podrían caracterizarse como esfuerzos para acabar con las funciones antagónicas que debe tener un multinodo, $\mathrm{y}$, en ese sentido, como una resistencia a la autoobservación. Según Adorno et. al. "la resistencia a la observación de uno mismo y la resistencia a comprender los hechos sociales son, en realidad, la misma cosa" (2006, 200). ¿Qué implican para el sistema cognitivo de segundo orden las acrobacias pseudocognitivas de los fundamentalistas? Desde el punto de vista de cerebros colectivos mayores, puede verse como una traba para participar en el llamado "cerebro mundial", en la medida en que se crea una especie de gueto o burbuja cognitiva. Y esa burbuja en última parte de un miedo a la apertura, a la posibilidad de replantearse la propia identidad. 
4. Segundo Rasgo del fundamentalismo: Burbujas cognitivas

Tanto Altemeyer (2006) como Schimmel (2008) notan los esfuerzos especiales que llevan a cabo los fundamentalistas para no exponerse a ideas que contradigan sus dogmas. Este esfuerzo es facilitado por los nuevos medios de comunicación, que hacen muy fácil que un individuo se exponga solamente a aquellas fuentes de información que confirman sus prejuicios, lo que termina fortaleciéndolos (ver Sunstein, 2003). En la medida en que el fundamentalista abraza narrativas marcadamente diferentes a las de la sociedad a la que pertenece, sus referentes históricos y culturales son tan diferentes a los del grupo exterior que la comunicación misma se hace imposible (ver Hedges, 2016, 163 sobre el caso del nacionalismo en la antigua Yugoslavia). Lo que es más preocupante, cuando a un fundamentalista se le presenta evidencia fuerte en contra de sus dogmas, lo más probable (por razones psicológicas) es que aumente su adhesión al dogma y redoble sus esfuerzos proselitistas (Schimmel, 2008, 175).

Si el fundamentalismo es un cierre cognitivo a ideas que vienen del exterior, entonces, en términos de un cerebro de segundo orden, se trata de una pérdida de conectividad entre los componentes del mismo. Ciertamente, las ideas fundamentalistas aportan variedad al cerebro mundial, pero al costo de una pérdida de cohesión. El fundamentalismo produciría un mal similar a la esclerosis múltiple, en que las diferentes partes del cerebro no se pueden comunicar entre sí. Dejo a juicio del lector si este es un cuadro exacto de nuestro mundo actual. He aquí, pues, el argumento, desde el punto de vista de la cognición colectiva, por el que debe rechazarse el fundamentalismo. Dicho rechazo no está dirigido a ningún contenido particular del fundamentalismo, sino a la forma en que opera la cognición en ciertos casos. Lo esencial del fundamentalismo es su rechazo a participar en el cerebro mundial, su baja capacidad de conectividad con otros.

En la medida en que se busca el fundamentalismo no en tal o cual postura o creencia, sino en este rasgo formal, podemos usarlo como rasero para evaluar si determinadas creencias son fundamentalistas. A continuación, examinaremos tres casos interesantes de fundamentalismo, con dos propósitos en mente: 1) ilustrar que el fundamentalismo puede encontrarse en formas de pensamiento lúcidas e ilustradas, y 2) mostrar que el elitismo es un rasgo esencial del fundamentalismo.

Steve Bannon, el estratega en jefe de Donald Trump y antiguo ejecutivo en jefe del portal de noticias de ultraderecha Breitbart, mediante referencias 
ocasionales, ha revivido el interés por Julius Evola, un pensador italiano que también ha sido recuperado por el movimiento griego "Amanecer Dorado" y por el movimiento nacionalista húngaro Jobbik, entre otras corrientes actuales de ultraderecha ( $c f r$. Horowitz, 2017). Evola es un tradicionalista que recupera la idea (presente en las mitologías griega, nórdica e hindú) de cuatro grandes edades: en el principio de un gran ciclo está la Edad de Oro, en que los hombres de las razas superiores controlan fuerzas divinas e instauran un orden perfecto en el que cada tipo de ser humano cumple su papel debido en una estructura jerárquica que, en su totalidad, es sacralizada por un contacto con lo divino; principalmente mediante la sublevación de las castas, se dan sucesivos pasos a edades inferiores, en que se pierde el contacto con lo divino y el orden trascendente que pone a cada cosa en su lugar (1995). La nuestra es la edad de Hierro o Kali Yuga, la época más baja: para Evola, la ciencia no es señal de nuestro progreso sino de nuestra degeneración y abandono de un conocimiento iniciático reservado para almas superiores a favor de un conocimiento puramente mecánico disponible para cualquiera $(1995,320)$.

No sorprende, pues, que Evola desdeñe el método científico y el método histórico. Para este pensador, los científicos que creen ser objetivos obran, sin saberlo, manipulados por las fuerzas anti-tradicionales de la edad de hierro (1995, xxxiii). No hay que poner atención a lo que dicen los historiadores de Roma, o los biógrafos académicos de Carlomagno: hay que atender a los mitos de fundación y a las sagas heroicas (1995, xxxiv). No se argumenta ni se ofrece prueba acerca del conocimiento tradicional: cuando uno se expone a él, o bien es un alma excelsa capaz de recordarlo, o está excesivamente condicionado por la edad moderna (1995, xxxiv). El conocimiento que pregona Evola no tiene nada que escuchar, ni nada que decirle, a otros tipos de saberes; y está disponible sólo para una minoría.

Sin duda, el pensamiento de Evola es extraño. ¿Es, no obstante, excepcional entre los fundamentalistas? Evola es elitista, ¿lo son los seguidores de Trump, que abrazan el antiintelectualismo, y el odio a las élites costeras de Los Ángeles y Nueva York? Si miramos el fundamentalismo religioso, podemos pensar que la doctrina de la revelación es una forma de elitismo, una justificación para cerrarse a voces que vengan por fuera del círculo de los elegidos, de los que han aceptado la revelación trascendente. El discurso de la nueva derecha de cuello azul es, ostensiblemente, anti-elitista en tanto antiintelectualista; pero en esencia rechaza a la ciencia y a la academia en nombre de algo superior: los real americans, el sentir del pueblo, y, en el peor de los casos, la causa de la raza blanca. 
Varios comentaristas políticos notaron la cantidad de discípulos del pensador Leo Strauss en el gabinete de George W. Bush, así como la aparente influencia de su filosofía en las decisiones tomadas por aquella administración: en particular, la justificación de invadir a Irak porque este país poseía armas de destrucción masiva, hoy ampliamente aceptada como sencillamente falsa, parece consistente con la lectura straussiana de la "mentira noble" platónica (p. ej., Shorris, 2004, Postel, 2003).

Como este pensamiento contrapone el filósofo a la multitud, éste, según Strauss y pensando en el ejemplo de Sócrates, debe protegerse de la multitud (López de Lizaga, 2007, 178): ésto lo consigue mediante una escritura opaca, que diga a las masas lo que éstas quieren escuchar, pero deje entrever a la élite, que sabe leer entre líneas, la verdad esotérica que se quiere transmitir (Strauss, 1996, pp. 57-92). Uno de los lugares en que se puede entrever más claramente el pensamiento de Strauss es en su comentario al Hierón de Jenofonte (Strauss, 2005): la mejor forma de gobierno es aquella en que un tirano tiene poder absoluto y es aconsejado por un filósofo capaz de ver las formas puras: esta forma de gobierno sería máximamente sabia, y a la vez flexible como no lo pueden ser las meras leyes abstractas (López de Lizaga, 2007, 179).

Según Strauss, la legitimidad política no proviene del consenso sino de la verdad, en el sentido platónico de acceso a las formas puras de lo real (en este caso, a las formas puras apropiadas a la ciencia política, como la forma del estado o de la justicia), acceso que sólo es posible para ciertos hombres con almas superiores (López de Lizaga, 2007, 175-182). En un cuerpo político en que haga falta el consenso del pueblo, las élites han de tomar sus decisiones de manera independiente, y luego resolver el problema técnico de cómo "vender" su decisión a las masas, convencerlas de lo ya determinado (y convencerlas de que fue su decisión); así pues, la justificación de invadir a Irak por poseer armas de destrucción masiva fue elegida por que se consideró que sería la más convincente para el pueblo (Shorris, 2004; ver también Gore, 2007, 116-118).

Ronald Reagan citó a Friedrich Hayek como uno de los pensadores que más influyeron en su gobierno, y éste fue un invitado especial de la Casa Blanca en varias ocasiones (Anderson, 1988, 164). La doctrina política y económica de Hayek fue acusada de sacralizadora del mercado por quienes detectan en ella un deslizamiento desde un modelo económico y un modelo de ser humano con propósito descriptivo a una utilización de ambos en forma prescriptiva, de modo que ya no se está ante una ciencia económica 
neutra sino ante un proyecto político radical (Vergara, 2005, 44-53; ver también Klein, 2007, p.61-68). Según los críticos, Hayek buscaba propagar un "pensamiento único" que "presenta como racional la creciente irracionalidad del sistema” (Vergara, 2005, 61). Sin embargo, Hayek fue miembro de la Sociedad Mont Pelerin, que tiene por meta la promoción de la libertad de pensamiento, los mercados libres y, en general, lo que Karl Popper (1981) llamaría la sociedad abierta.

Uno de los argumentos en contra de un estado fuerte en Camino de servidumbre es la tesis de que, en una democracia, tenderán a vencer el "común denominador más bajo”, la parte de la masa con las ideas más pobres intelectual y moralmente (Hayek, 1976, 170-177). Ahora bien, la desconfianza de Hayek en el criterio político de las masas cala más hondo: cuando Hayek propone su utopía política, propone "un sistema político bicameral, en el cual la primera cámara estaría formada por representantes políticos, los cuales designarían un equipo de gobierno que ejercería el poder ejecutivo (...) La segunda estaría compuesta por representantes que (...) serían elegidos entre los triunfadores (...)" (Vergara, 2005, 47). Por triunfadores hay que entender los triunfadores en el mercado, ya que Hayek identifica el éxito económico con la capacidad política (Vergara, 2005, 48). Podría interpretarse la utopía política de Hayek como el gobierno por parte del mercado libre, que sería coincidente con el gobierno por parte de la business class que entiende sus beneficios. En efecto:

Los ideales de la impersonalidad del mercado y del estado de derecho, según Hayek, sólo pueden lograrse si se "derroca la política"; entendida esta como un subsistema autónomo del económico (...). Dicho derrocamiento (...) requiere que una nueva élite reeemplace a la anterior, y ésta sería la neoliberal (...) Este es un partido transversal, una minoría consistente, cuyos miembros pertenecen a distintos partidos, a la administración del Estado (...) los medios comunicativos (...) y a diversas organizaciones (Vergara, 2005, 59).

¿Cómo puede "abolirse la política" desde el punto de vista del cerebro mundial? ¿No consiste en una cesación de la comunicación entre las neuronas, en nombre de un proyecto que se tiene por sí mismo como inconmoviblemente válido?

Así las cosas, vinculamos todo lo anteriormente dicho con otro rasgo del fundamentalismo que, incluso, puede verse como el fundamento de la manera de pensar autoritaria: una forma de emocionar difundida a través del discurso, aquella que está en la base de la aparente cohesión del grupo. Como 
parte final de este trabajo, introducimos la importancia de pensar las emociones como parte de los sistemas cognitivos de segundo orden.

\section{Tercer Rasgo del Fundamentalismo: Odio y emociones morales}

Humberto Maturana, pertenece junto con Beer, a ese grupo de pensadores de las organizaciones desde la teoría de sistemas. En su caso, le interesaban las organizaciones vivas, desde la más simple, una célula, hasta la más compleja, una social. No nos detendremos en las particularidades de su teoría, que comparte mucho con la de Beer, sino en un aspecto importante, que destacó en uno de sus libros, La democracia es una obra de arte (Maturana, 1995). En este libro señala que las emociones no son fenómenos abstractos, sino actividades cognitivas: por otro lado, ciertos emocionares permiten la apertura a significados del mundo social y material; significados que se comparten en la resonancia afectiva con los otros.

Así, para Maturana, hay emociones conducentes a organizaciones democráticas, es decir, a la apertura resonante con los afectos de otros, mientras que hay unas que conducen a organizaciones autoritarias, es decir, al cierre sobre sí mismas en esas burbujas cognitivas y elitismos de los que hemos hablado anteriormente.

Una perspectiva de las emociones (o de la actividad de emocionar siguiendo a Maturana) lleva a las consideraciones sobre el fundamentalismo a una dimensión que está a la base de los rasgos arriba mencionados. A su vez, implica pensar la cognición más allá del cerebro, como sugiere la perspectiva de cognición corporizada (por ejemplo, Gallagher y Zahavi, 2008). Para esta postura, la analogía con el cerebro, que nos permitió aquí entender los procesos cognitivos de segundo orden asemejándolos a lo que ocurre en la materia gris, es limitada. Y su limitación tiene que ver precisamente, con que la cognición humana no se limita al cerebro. Si bien cuando se alude a la figura de un "cerebro mundial" no se habla de un órgano compartido por el colectivo, sino de los procesos que emulan lo que ocurre a nivel neuronal, sacar la cognición del cerebro para trasladarla a todo el cuerpo también permite entender la cognición de los colectivos. Al final, los sistemas de segundo orden están compuestos por individuos corporizados y situados en un entorno material común.

En esa medida, los sesgos y heurísticos que un colectivo usa para resolver problemas también están vinculados a las experiencias de los cuerpos, a 
las coordinaciones y resonancias afectivas que se dan en la intersubjetividad. Aquí nos interesa particularmente cómo las emociones pueden estar involucradas en los procesos cognitivos, sobre todo aquellas asociadas al sentimiento de odio. E interesa que esas emociones no son solo sensaciones del cuerpo, sino, precisamente, actividades cognitivas como sugiere Maturana.

Haidt y Graham (2007), estudiaron las emociones vinculadas a la política, $y$ en lo que nos interesa, a pensamientos autoritarios. Ellos plantearon cinco fundamentos de la moral que, a su vez, están basados en emociones como la rabia, el asco, el desprecio, la gratitud y la vergüenza. Estos fundamentos, como su nombre indica, están a la base de todas las relaciones humanas porque determinan la manera de interrelacionarlos y los juicios que hacemos sobre otros. También son la base de la política; esto apoya la perspectiva de Maturana de que la cognición humana y la toma de decisiones sociales está basada en experiencias esencialmente afectivas.

Por ejemplo, pensemos en uno de los fundamentos de la moral de acuerdo con Haidt y Graham: el fundamento lealtad/traición. Esta díada está especialmente relacionada con los colectivos tribales y esos "cerebros" de segundo orden que caracterizan a los grupos autoritarios. Ella aparece en los discursos y propaganda política, y se basa en el maniqueísmo de que en el mundo hay buenos y malos. Pues bien, los buenos son los leales a la ideología y a los principios del grupo y los malos son aquellos que de alguna manera han traicionado dicha ideología y principios.

Este fundamento coincide con la dialéctica amigo/enemigo que Schmitt (2009) identifica con el autoritarismo, donde lo amigo es lo que está de acuerdo con los principios y la perpetuación de la tribu y enemigo es aquello que la amenaza. En el caso de los fundamentos de Haidt y Graham, el enemigo es el traidor y el amigo es el leal. Ahora bien, en un sistema que busca reducir la variedad sistémica a través del autoritarismo, el líder es el que decide quién es el enemigo y quién el amigo, a partir de la identidad del grupo, como es planteada de este modo jerárquico. En lo que interesa en este punto, esas categorías duales son altamente poderosas precisamente por lo reduccionistas que resultan (hay unos buenos y otros malos, y no puntos intermedios) por lo que son altamente efectivas en la reducción de la variedad, y porque están fundadas a su vez en emociones morales, que es lo que garantiza que a pesar de lo simples movilicen la acción, de acuerdo con lo que señalan Haidt y Graham.

En otras palabras, estas duplas son poderosas porque su significado emocional lleva efectivamente a la acción social. Y es que enemigo y amigo, leal 
o traidor, no son solamente marcos mentales de referencia es decir, términos que vinculan a los individuos con ideas, representaciones e imágenes que frecuentemente se difunden en la propaganda o en los discursos políticos (cf. Lakoff, 2008), sino que además están vinculados a emocionares de rechazo o de vínculo: el enemigo y el desleal, siguiendo a Haidt (2003), estarían asociados con emociones como asco, desprecio y rabia, y, por lo tanto, las acciones están encaminadas a apartarlo incluso agresivamente y el amigo con empatía y gratitud y por ende, buscan acercarlo.

Finalmente, el odio, como un sentimiento a su vez derivado de emociones como rabia y asco, de acuerdo con Haidt, es un sentimiento característico de los discursos autoritarios y fundamentalistas que resulta exitoso en su movilización del colectivo. Esto es así porque, según Ray, Mackie y Smith (2014) el odio termina asociándose a la identidad colectiva, lo que estaría de acuerdo con el propósito del subsistema 5 en el modelo de Beer. En el ejemplo, si el odio moviliza colectivamente es precisamente por estar asociado a una idea de identidad compartida, en donde el enemigo es el enemigo común, y no de un individuo. Ray, et. al. sugieren que el odio, y las movilizaciones en contra del enemigo, están asociadas a la victimización asociada con un clima emocional de miedo y angustia. Ese miedo y angustia, en lo que hemos visto aquí, no es más que el resultado de la insistencia en una identidad cerrada, encapsulada en esas burbujas con unos principios simples pero inamovibles. La simpleza y la caracterización de esos principios como necesarios para la supervivencia del grupo hace que los sentimientos de persecución sean constantes, y de ahí que el odio sea el sentimiento privilegiado. Es muy fácil que esas emociones negativas estén motivando la acción porque es muy frecuente enfrentarse a la amenaza en grupos que son ligeramente diferentes.

\section{CONCLUSIÓN}

¿Hasta qué punto se debe tolerar la intolerancia? Desde el punto de vista de la cognición colectiva, el problema es uno de conectividad. El fundamentalismo rehúsa a participar en el cerebro mundial; y un síntoma claro de ello es el elitismo, la idea de que la verdad sólo puede venir de una clase privilegiada. Frente al fundamentalismo, resultaría entonces crucial despersonalizar $y$ desustancializar la verdad, concebirla como aquello que fluye entre los nodos del multinodo, y no como aquello que es propiedad de algunos nodos en particular. Habría que reconocer y denunciar discursos que manifiestan una 
voluntad de no-participación en cerebros de segundo orden. Esto implica reconocer que, además de las falacias discursivas o los marcos de referencia, los discursos también suscitan emocionares que conducen a la acción. Esto involucra reconocer que un sistema cognitivo también se las ve con las corporalidades, y que las emociones son parte de la toma de decisiones políticas.

\section{REFERENCIAS}

Adorno, T.; Frenkel-Brunswik, E; Levinson, D.; y Sanford, N. (2006). “La Personalidad Autoritaria". Revista de Metodología de las Ciencias Sociales, 12, pp. 155-200.

Altemeyer, B. (2006). "The Authoritarians". https://drive.google.com/file/d/OBxxy 1K6fR81rckQxWi1hVFFRUDg/view? resourcekey=0-WGxUhtR8lhJdnFck4tTIyA (última consulta 10.06.2021).

Anderson, M. (1988). Revolution. San Diego. Harcourt Brace Jovanovich.

BeER, S. (1994). Designing Freedom. New York. John Wiley \& Sons.

BeER, S. (1995). Brain of the Firm. New York. John Wiley \& Sons.

Bula, G., Garavito, M. C y González, S. (2021). "Cognitive Bubbles: Towards a Logic of Fundamentalism”. Tópicos, 60, pp. 413-436.

ColombetTI, G. (2014). The Feeling Body: Affective Science Meets the Enactive Mind. Cambridge, MA, MIT Press.

DennetT, D. (2001). “Are we explaining consciousness yet?" Cognition, 79, pp. 221237

Dewey, J. (1950). "Democracy” en Randall, J. H. et. al. (eds) Readings in Philosophy. New York, Barnes and Noble.

EvolA, J. (1995). Revolt Against the Modern World. Rochester, VT. Inner Traditions

Gallagher, S. \& Zahavi, S. (2008). The phenomenological mind. London. Routledge.

GoRe, A. (2007). El ataque contra la razón. Barcelona. Random House.

GuIORA, A. (2014). Tolerating Intolerance: The Price of Protecting Extremism. Oxford. Oxford University Press.

HaIDT, J. (2003). “The Moral Emotions”, en R. Davidson, K. Scherer y H. Goldsmith (eds.), Handbook of Affective Sciences, pp. 852-870. Oxford. Oxford University Press.

HAIDT, J., \& Graham, J. (2007). "When morality opposes justice: Conservatives have moral intuitions that liberals may not recognize". Social Justice Research, 20, 98-116.

Halberstam, D. (1993). The Best and the Brightest. New York, Ballantine.

HayeK, F. (1976). Camino de Servidumbre. Madrid, Alianza.

Horowitz, J. (2017). Steve Bannon cited Italian thinker who inspired fascists. The New York Times, https://www.nytimes.com/2017/02/10/world/europe/bannon-vatican-julius-evola-fascism.html?_r=0 (última consulta 10.06.2021). 
MARÍA CLARA GARAVITO GÓMEZ Y GERMÁN BULA FUNDAMENTALISMO, ESTRATEGIAS ARGUMENTATIVAS Y EMOCIONES: UNA PERSPECTIVA DESDE LA COGNICIÓN DE SEGUNDO ORDEN

Janis, I. (1980). “Groupthink”, en Leavitt, H., Pondit, L. y Boje, M. Readings in Managerial Psychology. Chicago, University of Chicago Press.

KleIN, M. (2007). The Shock Doctrine. Nueva York: Picador.

Lakoff, G. (2007). No pienses en un elefante. Lenguaje y debate politico. Madrid: Editorial Complutense.

López de Lizaga, J. (2007). “Leo Strauss y la rehabilitación de la filosofía política clásica”. Studiom, Revista de humanidades, 13, pp. 171-184.

Maturana, H. (1995). La democracia es una obra de arte. Bogotá, Magisterio.

REYNOLDS, C. (1987). "Flocks, herds and schools: a distributed behavioral model. SIGGRAPH '87". Proceedings of the 14th annual conference on Computer graphics and interactive techniques pp. 25-34. http://dl.acm.org/citation. cfm?id=37406 (última consulta 10.06.2021).

Page, S. (2007a). "Making the Difference: Applying a Logic of Diversity”. Academy of Management Perspectives, 21(4), pp. 6-20.

Page, S. (2007b). The difference. Princeton, NJ, Princeton University Press.

Popper, K. (1981). La sociedad abierta y sus enemigos. Barcelona, Paidós.

Postel, D. (2003). "Noble lies and perpetual war: Leo Strauss, the neocons, and Iraq”. openDemocracy.net, https://www.opendemocracy.net/faithiraqwarphiloshophy/article_1542.jsp (última consulta 10.06.2021).

Ray, D. G., Mackie, D. M., y Smith E. R. (2014). "Intergroup emotion: self-categorization, emotion, and the regulation of intergroup conflict”, en von Scheve, C. y Salmela, M. (eds). Collective Emotions Perspectives from Psychology, Philosophy, and Sociology. Oxford, Oxford University Press.

Schimmel, S. (2008). The Tenacity of Unreasonable Beliefs. Oxford: UK. Oxford University Press.

Schuler, D. (2001). “Cultivating Society’s Civic Intelligence: Patterns for a New 'World Brain'”. Information, Communication E Society, 4:2 pp. 157-181.

SHORRIS, E. (2004). Ignoble liars: Leo Strauss, George Bush, and the philosophy of mass deception. Harper's Magazine, 1, pp. 65-71.

Schmitt, C. (2009). El concepto de lo político. Madrid, Alianza editorial.

STRAuss, L. (1996). Persecución y el arte de escribir. Valencia, Alfons el Magnánim.

STRAuss, L. (2005). Sobre la tiranía. Madrid, Encuentro.

Sunstein, C. (2003). República.com. Barcelona, Paidós.

Vergara, J. (2005). La utopía neoliberal y sus críticos. Utopía y praxis latinoamericana, 10, 31, pp. 37-62. 